\title{
Recombinant Moraxella bovoculi cytotoxin-ISCOM matrix adjuvanted vaccine to prevent naturally occurring infectious bovine keratoconjunctivitis
}

\author{
John A. Angelos • V. Michael Lane • Louise M. Ball • \\ John F. Hess
}

Accepted: 18 February 2010 /Published online: 10 March 2010

(C) The Author(s) 2010. This article is published with open access at Springerlink.com

\begin{abstract}
A randomized, blinded, controlled field trial was conducted during summer 2006 in a northern California, USA, herd of beef cattle to evaluate the efficacy of a recombinant Moraxella bovoculi cytotoxin subunit vaccine to prevent naturally occurring infectious bovine keratoconjunctivitis (IBK; pinkeye). A convenience sample comprised of 127 steers were administered a subcutaneous dose of either adjuvant alone (ISCOM matrices; control group) or recombinant $M$. bovoculi cytotoxin carboxy terminus adjuvanted with ISCOM matrices (MbvA group) and were boostered 21 days later. The steers were examined once weekly for 15 weeks for evidence of IBK. No significant difference in the cumulative proportion of corneal ulcerations was detected between groups. Compared to the control calves, the MbvA vaccinates had significantly higher increases in serum neutralizing titers to $M$. bovoculi hemolysin between week 0 and week 6 . The prevalence of M. bovis isolations was higher from ulcerated eyes of calves vaccinated with MbvA as compared to control calves. Vaccination of calves against the carboxy terminus of M. bovoculi RTX toxin resulted in significant increases in serum hemolysin neutralizing titers and may modulate organism type cultured from ulcerated eyes of calves in herds where both $M$. bovis and $M$. bovoculi exist. Use of $M$. bovoculi antigens alone in vaccines to prevent IBK may not be beneficial in herds where IBK is associated with both M. bovoculi and M. bovis.
\end{abstract}

J. A. Angelos $(\bowtie) \cdot$ L. M. Ball

Department of Medicine and Epidemiology, University of California, 2108 Tupper Hall, Davis, CA 95616, USA

e-mail: jaangelos@ucdavis.edu

V. M. Lane

Department of Population Health and Reproduction, University of California, Davis, CA 95616, USA

J. F. Hess

Department of Cell Biology and Human Anatomy, School of Medicine, University of California, Davis, CA 95616, USA

Present Address:

L. M. Ball

Department of Microbiology, University of Virginia Health Sciences Center, Box 800734,

Jordan Hall, Room 7022, Charlottesville, VA 22908, USA 
Keywords Moraxella bovoculi $\cdot$ Moraxella bovis - Cytotoxin · Subunit ·

Infectious bovine keratoconjunctivitis

\section{Introduction}

Infectious bovine keratoconjunctivitis (IBK; pinkeye) is the most common eye disease of cattle and is seen worldwide affecting both dairy and beef breeds of cattle. The clinical signs of IBK include corneal ulceration, corneal edema, ocular pain, photophobia, and lacrimation. In the worst cases the cornea can rupture leading to permanent blindness. In 1960 the Gram negative rod Moraxella bovis (M. bovis) was demonstrated to be an etiologic agent of IBK (Henson and Grumbles 1960). In addition to M. bovis, however, Gram negative cocci have been isolated with or without M. bovis from cattle with IBK (Elad et al. 1988; Fairlie 1966; Pugh and Hughes 1971; Spradbrow 1967; Wilcox 1970). Prior to 2007, Gram negative cocci isolated from eyes of cattle with IBK were likely to be identified as Moraxella ovis, Moraxella ovis-like, Branhamella ovis, or Branhamella ovis-like. The isolation and characterization of a novel species of Moraxella designated Moraxella bovoculi (M. bovoculi) from ulcerated eyes of beef and dairy calves with IBK has raised the question whether $M$. bovis is the only organism that causes IBK (Angelos et al. 2007c).

To date, only isolated studies have reported on the prevalence of $M$. bovoculi as compared to $M$. bovis in IBK-associated corneal ulcers. In one study, bacteria that were cultured from IBK-associated corneal ulcers were identified as Gram negative cocci in $\sim 46 \%$ of ulcers, while only $\sim 21 \%$ of ulcers were $M$. bovis positive (Dueger et al. 2004). Gram negative cocci from that study were subsequently identified as M. bovoculi (Angelos et al. 2007c). In another study $M$. bovoculi without $M$. bovis was isolated from $\sim 51 \%$ of initial IBK-associated corneal ulcers; M. bovis without M. bovoculi was isolated from $20 \%$ of initial ulcers in that trial (Angelos et al. 2007b). Moraxella bovoculi has also been isolated from reindeer with infectious keratoconjunctivitis (Tryland et al. 2009). Despite the presence of M. bovoculi in IBK-associated ulcers, however, Koch's postulates have not been established for M. bovoculi and IBK. In a recently published randomized and blinded field trial, an M. bovoculi autogenous bacterin was not effective against IBK (Funk et al. 2009). In that study the commercially prepared $M$. bovoculi bacterin was composed of 2 isolates made during the preceding year from the same herd. There are, however, anecdotal reports of reduced IBK in herds previously vaccinated with $M$. bovis bacterins when a switch to an $M$. bovoculi bacterin is made or when an $M$. bovoculi bacterin is used in conjunction with an $M$. bovis bacterin.

Essential proteins of $M$. bovis that are necessary for pathogenicity include pilin and cytotoxin (hemolysin, cytolysin). Pili enable M. bovis to adhere to the corneal surface (Annuar and Wilcox 1985; Moore and Rutter 1989; Ruehl et al. 1993a). The M. bovis cytotoxin lyses bovine neutrophils, erythrocytes, lymphocytes, and corneal epithelial cells leading to corneal ulceration (Beard and Moore 1994; Gray et al. 1995; Hoien-Dalen et al. 1990; Kagonyera et al. 1989a; Kagonyera et al. 1989b). The M. bovis cytotoxin (MbxA) is an RTX (repeats in the structural toxin) toxin (Angelos et al. 2001) that resides within an RTX operon on a pathogenicity island in M. bovis (Angelos et al. 2003; Hess and Angelos 2006). The $M$. bovis cytotoxin is conserved between geographically diverse strains (Angelos and Ball 2007). Moraxella bovoculi possesses a complete RTX operon that encodes an RTX protein designated MbvA, which has 83\% deduced amino acid sequence identity with MbxA (Angelos et al. 2007a).

Previous studies have examined the efficacy of partially purified native $M$. bovis cytotoxin or recombinant $M$. bovis cytotoxin to prevent IBK. A partially purified $M$. bovis cytolysin 
(cytotoxin) adjuvanted with Quil A was effective at reducing IBK in vaccinated calves (George et al. 2005). Limitations to large scale production of this partially purified M. bovis cytolysin vaccine prompted further investigations into recombinant $M$. bovis cytotoxin vaccines. In one trial the carboxy terminus of MbxA served as the vaccine antigen (Angelos et al. 2004); in a subsequent trial, the same carboxy terminus of MbxA was expressed as a fusion protein with the amino terminus of M. bovis pilin (Angelos et al. 2007b). In these trials, reduced occurrences of IBK amongst vaccinates as compared to control calves were reported, however, the differences between vaccinates and controls were not consistently significant. The presence of $M$. bovoculi in cultures of eyes from IBK affected calves was documented in the 2007 trial and may have negatively affected vaccine efficacy, given that both M. bovis and $M$. bovoculi were present in the test herd. The present study was designed to evaluate the efficacy of recombinant $M$. bovoculi MbvA to prevent naturally occurring IBK.

\section{Materials and methods}

\section{Study population}

The study population was a convenience sample of 127 Angus $(n=68)$, Hereford $(n=2)$, Angus-Hereford cross $(n=42)$, and Hereford cross $(n=15)$ calves aged 4.9-7.1 months old. Weaning weights of the calves ranged from 159 to $294 \mathrm{~kg}$. The calves were maintained on mixed grass pastures and were managed as a single cohort at the University of California Sierra Foothills Field Station, Brown's Valley, CA, USA. The terrain consisted of Sierra foothill range pasture that varied from 200-400 meters above sea level. The herd historically had a high prevalence of IBK of approximately $50 \%$ of calves. The trial started on May 2, 2006 and ended on August 15, 2006. Neither the steers nor dams of these steers had been vaccinated against IBK. The experimental procedure was approved by the University of California Institutional Animal Care and Use Committee (\# 06-12522).

Pre-enrollment examination and vaccination

Calves were restrained in a hydraulic squeeze chute and each eye was examined grossly for evidence of preexisting corneal disease. Calves with two normally appearing corneas were randomly assigned to either a control or vaccine group by use of a blocked randomization scheme. On the day of enrollment (day 0) and on day 21 when booster vaccinations were administered, calves received $2 \mathrm{ml}$ of either immune stimulating complex (ISCOM) matrix adjuvant (control group) or recombinant $M$. bovoculi cytotoxin carboxy terminus adjuvanted with ISCOM matrix (MbvA; vaccine group). The study personnel were blinded as to the contents of the vaccines that were administered. In addition, on day 0 all calves were vaccinated against respiratory viral pathogens (Bovi-Shield ${ }^{\circledR}$ Gold 5, Pfizer Animal Health, New York, NY, USA), and on day 14 all calves received a multivalent Clostridium-Mannheimia vaccine (One Shot Ultra ${ }^{\mathrm{TM}}$ 8; Pfizer Animal Health), selenium supplementation (Mu-Se ${ }^{\circledR}$, Schering-Plough, Union, NJ, USA), and were dewormed (Dectomax ${ }^{\circledR}$ Pour-On, Pfizer Animal Health).

Post-enrollment examination

Once weekly for 15 weeks each calf was restrained in a hydraulic squeeze chute and both eyes were examined grossly for the presence of corneal opacification by one of two examiners blinded as to the vaccine type that calves had been administered. If corneal 
opacification was observed, the inferior subconjunctival cul-de-sac was swabbed with a sterile Dacron swab. Such eyes were then stained with fluorescein (Sigma, St. Louis, MO, USA) and examined with a flashlight to determine if a corneal ulcer was present; if so, the swabs were further processed as described below. A corneal ulcer was considered to be associated with IBK unless it appeared to be mechanically induced (i.e., the pattern of fluorescein staining was linear or stellate), or was present in an eye from which a plant awn was removed. If a mechanically induced ulcer was still present at the next weekly observation, it was considered to be associated with IBK, and in such cases, that observation day was counted as the starting day for the ulcer. Corneal ulcer scores (CUS) were assigned according to the widest ulcer diameter as measured with a ruler held next to the affected eye: $0=$ no ulcer; $1=$ less than or equal to $5 \mathrm{~mm}$; $2=$ greater than $5 \mathrm{~mm}$; and 3 =perforated corneal ulcer. Eyes with ulcers were digitally photographed (Canon EOS D30, Canon U.S.A., Inc., Lake Success, NY, USA) with a ruler held next to the eye for subsequent determination of the corneal ulcer surface area as described below. Calves with a CUS of 2 or greater were treated once with florfenicol $(40 \mathrm{mg} / \mathrm{kg}$ subcutaneously; Nuflor ${ }^{\circledR}$, Schering-Plough Animal Health). Rubber gloves/aprons were worn by examiners and were rinsed with $1 \%$ chlorhexidine solution between calves to prevent iatrogenic ocular infections.

\section{Bacterial culturing of ulcerated eyes}

Ocular swabs collected from eyes of calves with confirmed ulcers were streaked onto 5\% sheep blood agar plates that were stored at $4^{\circ} \mathrm{C}$ during transport back to the laboratory; these plates were subsequently streaked for isolation. Plates were incubated at $35^{\circ} \mathrm{C}$ for 24 hour and examined for the presence of beta hemolysis. Beta hemolytic colonies were subcultured and identified using biochemical criteria established for M. bovis (Weyant et al. 1996) or M. bovoculi (Angelos et al. 2007c).

\section{Vaccine formulation}

Growth and propagation conditions of $M$. bovoculi $237\left(\right.$ ATCC BAA-1259 ${ }^{\mathrm{T}}=\mathrm{CCUG}$ $52049^{\mathrm{T}}$ ) were performed as previously described (Angelos et al. 2007a). The methods for cloning and expressing the carboxy terminus (amino acids 590 through 927) of MbvA were as described (Angelos et al. 2007a). The recombinant carboxy terminus of MbvA was purified using a protocol for purifying recombinant M. bovis cytotoxin (Angelos et al. 2003). The final MbvA vaccine was mixed 1:1 with ISCOM matrices that had been prepared as previously described (Angelos et al. 2004) such that the final vaccine delivered $500 \mu \mathrm{g}$ of recombinant protein in a $2 \mathrm{ml}$ dose. The ISCOM matrix solution was mixed 1:1 with phosphate buffered saline (PBS; $\mathrm{pH} 7.4$ ) to prepare the final $2 \mathrm{ml}$ control vaccine dose. All vaccinations were administered subcutaneously in the neck.

\section{Corneal ulcer surface area measurements}

Corneal ulcers in the digital images of ulcerated eyes were traced on a computer tablet (Wacom Cintiq 15X LCD tablet, Wacom Technology Corporation, Vancouver, WA,USA) using public domain image analysis software (ImageJ program; available at http://rsbweb. nih.gov/ij). Magnification differences between photographs were accounted for by standardizing the scale of each tracing using the ruler in each photograph. The mean of 3 tracings of each ulcer was used to calculate the corneal ulcer surface area measurement; for data analysis, the square root of the corneal ulcer surface area was used to represent the 
ulcer surface area measurement (SAM). The limit of detection was $0.008 \mathrm{~cm}^{2}$, an area corresponding to a $1 \mathrm{~mm}$ diameter circle.

\section{Hemolysis neutralization assay}

Whole blood was collected by jugular venipuncture into serum separator tubes (Corvac tubes, Sherwood-Davis \& Geck, St. Louis, MO, USA) on day 0, day 42, and day 105. Serum was harvested and stored at $-20^{\circ} \mathrm{C}$. Prior to use in hemolysis neutralization assays, serum was heat inactivated at $56^{\circ} \mathrm{C}$ for 1 hour. Native cytotoxin in diafiltered retentate derived from culture supernatant of M. bovoculi 237 was prepared using a previously described method for preparation of $M$. bovis cytotoxin (Angelos et al. 2001). The diafiltered retentate containing native $M$. bovoculi cytotoxin was stored at $-80^{\circ} \mathrm{C}$ until use in the hemolysis neutralization assays which were performed as previously described (Angelos et al. 2007b). The final dilution of $M$. bovoculi 237 diafiltered retentate in the neutralization assays was 1:16. The dilution endpoint was defined as the last dilution at which no hemolysis was observed. The geometric mean of three sample endpoints was used as the final dilution endpoint. For statistical analyses, the day 0-42 and day 0-105 changes in serum hemolysin neutralizing titers of nonulcerated calves to native M. bovoculi 237 cytotoxin were evaluated.

\section{Statistical analyses}

Baseline differences between groups with respect to age at enrollment/weight at weaning/ day 0 serum hemolysin neutralizing titer and breed were evaluated by the Mann-Whitney and Pearson chi square tests, respectively. The primary outcome variable that was used to evaluate vaccine efficacy was the occurrence of IBK in calves as measured by the cumulative proportion of calves ulcerated at weeks 8 and 15 (cumulative proportion ulcerated). Additional variables examined included the proportion of calves that required at least one florfenicol treatment; the median number of days until the first ulcer was observed; the median surface area measurement of the first ulcer (median SAM for first ulcer); the median peak surface area measurement of the first ulcer (median peak SAM for first ulcer); and the median number of days for the first ulcer to heal (CUS=0; median days to heal for first ulcer). Differences in the cumulative proportion of calves that developed corneal ulcers and the number of treatments for IBK were evaluated using Pearson chisquare analysis. Risk ratios with $95 \%$ confidence intervals (CI) for developing a first ulcer were calculated for the adjuvant group versus the MbvA group at weeks 8 and 15. The Mann-Whitney test was used to evaluate differences between groups with respect to median days to first ulcer, median SAM for the first ulcer, median peak SAM for the first ulcer, median days to heal for the first ulcer, and changes in day 0-42 and day 0-105 serum hemolysin neutralizing titers. To reduce possible influences of acquired immunity due to the development of IBK, changes in hemolysin neutralizing titers were evaluated in calves that remained nonulcerated through day 105. A Kaplan-Meier (KM) survival curve was constructed for the time to first ulcer occurrence; differences between KM curves were evaluated using the log-rank test. Time to first ulcer was defined as the time of enrollment (day 0) to the time of first observed IBK-associated ulcer (CUS $>0$ ). Prevalence ratios for the isolation of $M$. bovis and M. bovoculi were calculated for the MbvA group versus the adjuvant group. In all comparisons, a value of $P<0.05$ was considered significant; a $95 \%$ CI that did not include 1.0 was considered significant. Statistical analyses were performed with SPSS (version 16.0; SPSS Inc., Chicago, IL, USA), and StatCalc (version 3.5.1; EpiInfo; available at: http://www.cdc.gov/epiinfo/). 


\section{Results}

Verification of randomization

No significant differences were detected between the groups with respect to age at enrollment, calf weaning weight, day 0 serum hemolysin neutralizing titer, and breed: $P=$ $0.63, P=0.142, P=0.91$, and $P=0.64$, respectively.

\section{Efficacy of recombinant MbvA vaccine to prevent IBK}

At week 8 , the cumulative proportion of ulcerated calves was $0.4(25 / 62)$ and $0.37(24 / 65)$ in the control and MbvA groups, respectively. At week 15, the cumulative proportion of ulcerated calves was 0.52 (32/62) and 0.45 (29/65), in the control and MbvA groups, respectively. The differences in the cumulative proportion of ulcerated calves at week 8 and week 15 were not significantly different $(\mathrm{P}=0.69$ and 0.43 , respectively; Table 1$)$. The risk for ulcer development in control calves by week 8 was 1.09 times more than that of the MbvA group calves; $95 \%$ CI $(0.70,1.69)$. The risk for ulcer development in control calves by week 15 was 1.16 times more than that of the MbvA group calves; $95 \%$ CI $(0.81,1.66)$. No significant differences between vaccine and control groups were observed with respect to the proportion of calves requiring at least one nuflor treatment, median SAM of the first ulcer, median peak SAM for the first ulcer, median days to heal for the first ulcer, or the median change in weight from weaning to day 105 (Table 1). Kaplan-Meier survival analysis did not show a significant difference between groups when the times to first ulcer were examined $(\mathrm{P}=0.38)$ (Fig. 1$)$.

Systemic immune response following vaccination

The median change in the day 0-42 serum hemolysin neutralizing titers of nonulcerated calves was significantly higher in the MbvA group (4.3) versus the control group (0) $(P=$ 0.004 ) (Table 2). There was no significant difference in the median change in the day $0-105$ serum hemolysin neutralizing between nonulcerated calves in the control and MbvA groups (median $=0$ for both groups).

Bacterial isolates from initial ulcers

From 61 initial ulcers that developed in calves of the combined vaccine groups, M. bovis and $M$. bovoculi were both isolated, however, the predominant organism isolated from initial ulcers was M. bovis (44 isolations) as compared to M. bovoculi (35 isolations) (Table 3). Moraxella bovis was 2.88 times more likely to be isolated from ulcers in calves from the MbvA group than from calves in the control group $(\mathrm{P}=0.09$; Table 4).

\section{Discussion}

During this 15 week trial, significant differences in the cumulative proportion of calves that developed corneal ulcerations were not detected between the group that received a recombinant $M$. bovoculi cytotoxin subunit vaccine and a control group of calves that received the adjuvant (ISCOM) alone. It is possible that a recombinant $M$. bovoculi cytotoxin vaccine might be more effective in herds in which a majority of IBK cases are 
Table 1 Vaccine group outcomes assessing vaccine efficacy

\begin{tabular}{|c|c|c|}
\hline \multirow[t]{2}{*}{ Outcome } & \multicolumn{2}{|l|}{ Group $^{\mathrm{a}}$} \\
\hline & Control & MbvA \\
\hline cumulative proportion ulcerated wk 8 & 0.40 & 0.37 \\
\hline number of calves ulcerated & 25 & 24 \\
\hline$P^{\mathrm{b}}$ & & 0.69 \\
\hline cumulative proportion ulcerated wk 15 & 0.52 & 0.45 \\
\hline number of calves ulcerated & 32 & 29 \\
\hline$P$ & & 0.43 \\
\hline proportion requiring at least 1 florfenicol treatment $\mathrm{c}^{\mathrm{c}}$ & 0.37 & 0.34 \\
\hline total number of calves treated & 23 & 22 \\
\hline$P$ & & 0.70 \\
\hline median days to first ulcer (min.- $\max$.) & $42(28-98)$ & $42(28-77)$ \\
\hline $\mathrm{N}$ & 32 & 29 \\
\hline$P$ & & 0.70 \\
\hline median SAM (min.--max.) for first ulcer & $0.32(0.11-1.55)$ & $0.34(0.11-1.22)$ \\
\hline $\mathrm{N}$ & 32 & 29 \\
\hline$P$ & & 0.97 \\
\hline median peak SAM (min.--max.) for first ulcer & $0.19(0.11-0.46)$ & $0.16(0.11-0.4)$ \\
\hline $\mathrm{N}^{\mathrm{d}, \mathrm{e}}$ & 11 & 8 \\
\hline$P$ & & 0.90 \\
\hline median days (min.- $\max$.) to heal for first ulcer & $7(6-21)$ & $7(7-14)$ \\
\hline $\mathrm{N}^{\mathrm{e}}$ & 11 & 8 \\
\hline$P$ & & 0.57 \\
\hline $\begin{array}{l}\text { median weight change (min.- } \max .) \text { weaning to day } \\
105\end{array}$ & $31.4(13.6-58.2)$ & $29.1(5.5-56.4)$ \\
\hline $\mathrm{N}$ & 62 & 65 \\
\hline $\mathrm{P}$ & & 0.42 \\
\hline
\end{tabular}

\footnotetext{
${ }^{\text {a }}$ Sample size: control, 62; MbvA, 65

${ }^{\mathrm{b}} P$ values reflect comparisons with the control group

${ }^{c}$ expressed as total number calves receiving at least 1 florfenicol treatment/number calves in group

${ }^{\mathrm{d}}$ peak first ulcer surface areas could not be calculated for 1 calf due to missing photographs

e excludes treated calves
}

associated with $M$. bovoculi relative to $M$. bovis. The sample size in this study was determined by the availability of steers, and it is possible that a larger sample size may have increased the chances of finding a significant difference. In order to control for environmental variables such as plant awns, face flies, and UV radiation that can predispose to IBK, the control calves and vaccinated calves were pastured as a single group. Herd level immunity amongst the MbvA vaccinated group, however, may have lowered the power of this study as the occurrence of IBK in the control calves may have been reduced.

The fundamental question of whether $M$. bovoculi is a primary pathogen in IBK has not been answered, and specific conclusions regarding the effectiveness of including recombinant $M$. bovoculi antigens in vaccines to prevent IBK remain speculative. The use of a $M$. bovoculi autogenous vaccine was recently shown to be ineffective at preventing 


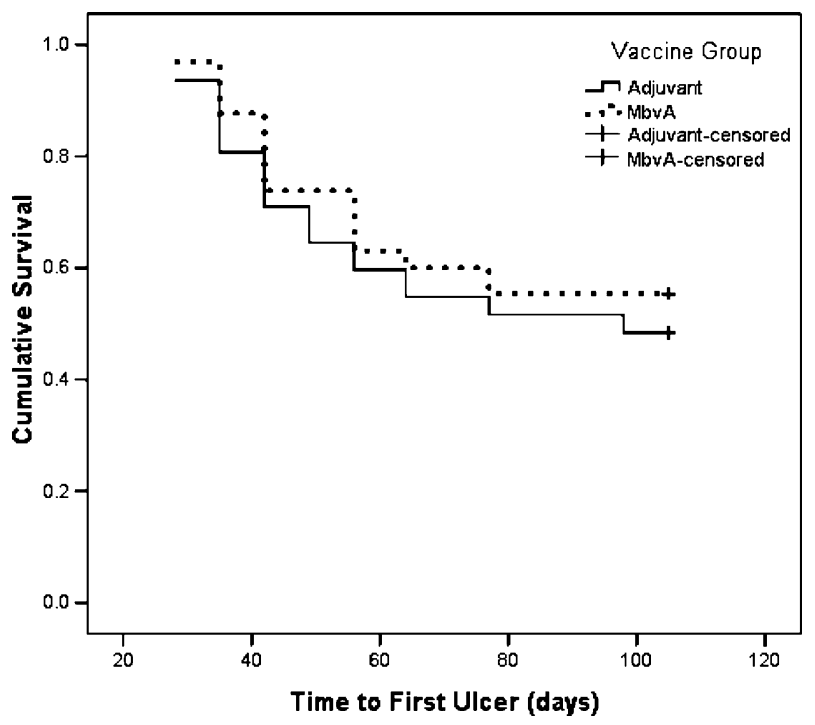

Fig. 1 Kaplan-Meier survival function curves of time to first corneal ulcer attributed to infectious bovine keratoconjunctivitis in cross-bred beef steers vaccinated with a recombinant Moraxella bovoculi cytotoxin vaccine $(M b v A ; n=65)$ or adjuvant alone (control; $n=62)$

naturally occurring IBK (Funk et al. 2009). In that study the experimental vaccine was a commercially prepared bacterin composed of two different $M$. bovoculi strains. It is possible that limited cross reactivity between the vaccine strains and $M$. bovoculi in the test herd during the trial period may have reduced the efficacy of the autogenous bacterin; cell surface antigens such as pili are considered important determinants in cross reactivity and protection against M. bovis (Lepper 1988). There are, however, anecdotal reports of autogenous $M$. bovoculi bacterins preventing IBK in herds when traditional M. bovis vaccines are not effective. These reports coupled with the occurrences of corneal ulcers from which only $M$. bovoculi can be isolated, as occurred in the present study as well as a previous study (Angelos et al. 2007b), support a hypothesis that M. bovoculi is important in IBK pathogenesis. If true, this hypothesis could support the rationale to include $M$. bovoculi antigens in IBK vaccines, especially in those herds where IBK has been associated with $M$. bovoculi.

Table 2 Median (min.-max.) changes from day $0-42$ and 0-105 in serum hemolysin neutralizing titers of nonulcerated calves to native $M$. bovoculi cytotoxin

\begin{tabular}{lll}
\hline & \multicolumn{2}{l}{ Vaccine group } \\
\cline { 2 - 3 } & control $(\mathrm{N}=30)$ & MbvA (N=36) \\
\hline Day 0-42 & $0.0(-12.0-10.3)$ & $4.3(-12.0-28.0)$ \\
$P$ a & & 0.004 \\
Day 0-105 & $0.0(-8.0-12.0)$ & $0.0(-16.0-12.0)$ \\
$P$ & & 0.608
\end{tabular}

${ }^{\text {a }} \mathrm{P}$ value reflects the comparison with the adjuvant control group using median values (Mann-Whitney test) 
Table 3 Frequency of Moraxella spp. isolations from 61 initial ulcers

\begin{tabular}{lccr}
\hline Organism & \multicolumn{2}{c}{ Vaccine group } & Total \\
\cline { 2 - 4 } & control $(\mathrm{N}=32)$ & MbvA (N=29) & \\
\hline M. bovoculi & 9 & 3 & 12 \\
M. bovis & 11 & 10 & 21 \\
M. bovoculi plus $M$. bovis & 9 & 14 & 23 \\
No Moraxellae isolated & 3 & 2 & 5 \\
\hline
\end{tabular}

The recombinant $M$. bovoculi antigen that was used in this trial represented the carboxy terminal 338 amino acids of MbvA; in total, $m b v A$ encodes a 927 amino acid protein (Angelos et al. 2007a). Previously, the analogous portion of M. bovis MbxA was demonstrated to elicit a hemolysis neutralizing antibody response in rabbits (Angelos et al. 2007a). Based on the results of the present study, it can be concluded that calves respond similarly against MbvA by mounting a hemolysis neutralizing antibody response. The increase in neutralizing titer between day 0-42 was significantly higher in nonulcerated vaccinated calves compared to nonulcerated control group calves. The significant increase, however, was not maintained for the duration of the trial. Previously when we measured the change in serum hemolysis neutralizing titer of non-ulcerated calves that were vaccinated with a recombinant $M$. bovis pilin-cytotoxin, we identified statistically significant increases not only in the day 0 to day 42 ( 3 weeks post booster vaccination) titers, but also in the day 0 to day 126 titers (Angelos et al. 2007b). It is possible that the observed difference in the duration of immune responsiveness against MbvA versus MbxA in our two studies is related to the composition of the antigen used in the neutralization assays. In a previous study that examined the stability of $M$. bovis cytotoxin (MbxA) prepared as a diafiltered retentate derived from culture supernatant, both full length MbxA as well as immunoreactive MbxA fragments were identified in the diafiltered retentate (George et al. 2004). It is possible that during the preparation of $M$. bovoculi diafiltered retentate used for the hemolysis neutralization assays, partial length fragments of MbvA were similarly copurified along with native MbvA, and that in the neutralization assays, these partial length fragments, while lacking hemolytic activity, still bound to antibodies against MbvA and rendered such antibodies unavailable to neutralize full-length native MbvA. It is also possible that other proteins copurified with MbvA in the diafiltered retentate of M. bovoculi culture supernatant may have served to promote hemolysis, effectively reducing the measured titers. In the hemolysis neutralizing assays that were performed in the present study, the $M$. bovoculi diafiltered retentate had less hemolytic activity relative to similarly prepared M. bovis diafiltered retentate that we have prepared for previous studies; as such,

Table 4 Frequency of isolation of $M$. bovis and prevalence ratio (PR) for the association of M. bovis with initial ulcers

\begin{tabular}{|c|c|c|c|c|}
\hline \multirow[t]{2}{*}{ M. bovis isolated from ulcer } & \multicolumn{2}{|c|}{ Vaccine group } & \multirow[t]{2}{*}{ PR } & \multirow[t]{2}{*}{$P$} \\
\hline & MbvA & control & & \\
\hline Yes & 24 & 20 & 2.88 & 0.094 \\
\hline No & 5 & 12 & & \\
\hline Total & 29 & 32 & & \\
\hline
\end{tabular}


the working dilution of the $M$. bovoculi diafiltered retentate in the neutralization assays was only $1: 16$. In contrast, the final working dilution of $M$. bovis diafiltered retentate in the neutralization assays in our 2007 trial was 1:128 (Angelos et al. 2007b).

It is possible that an immune response against epitopes found in the amino terminus of the $M$. bovoculi cytotoxin might improve the efficacy of a recombinant $M$. bovoculi cytotoxin vaccine. While no previous studies have examined the effectiveness of full length recombinant $M$. bovis cytotoxin to stimulate a hemolysis neutralizing immune response, one study identified the presence of hemolysis neutralizing epitopes in the amino terminus of HlyA, the RTX toxin expressed by uropathogenic Escherichia coli (Ji and O'Hanley 1990). As such, a full length recombinant M. bovoculi and/or M. bovis cytotoxin may improve the efficacy of recombinant Moraxella spp. cytotoxin based vaccine against IBK as compared to carboxy terminus cytotoxin based vaccines as reported here and in earlier studies (Angelos et al. 2007b; Angelos et al. 2004).

In this study, the prevalence of $M$. bovis from cultures of initial ulcers was nearly three times higher in the MbvA vaccinates compared to the control calves. While this higher prevalence was not statistically significant, it may have biological significance. In a previous study that evaluated a recombinant $M$. bovis pilin-cytotoxin vaccine, we found a higher prevalence of $M$. bovoculi in cultures from initial ulcers of calves vaccinated with two M. bovis antigens: pilin and cytotoxin (Angelos et al. 2007b). Moreover, there was a higher prevalence of $M$. bovis in ocular cultures from control calves as compared to $M$. bovis pilin-cytotoxin vaccinated calves (Angelos et al. 2007b). In the present study it is possible that the immune response against $M$. bovoculi MbvA allowed M. bovis to colonize the ocular surface more readily or provided some other competitive advantage relative to $M$. bovoculi. The potential for a host immune response to alter the expression of different $M$. bovis pili was previously suggested (Ruehl et al. 1993b). Additional investigations of the ocular flora of not only IBK-affected, but also normal healthy eyes of calves are necessary to help define mechanisms by which vaccination may affect the development of IBK and of the role that $M$. bovoculi plays in IBK pathogenesis.

Acknowledgements This study was funded by UC Davis School of Veterinary Medicine Center for Food Animal Health Funds and Formula Funds. Technical assistance from Natalie S. Tankersley and Diane M. Rhodes with data collection and from Dr. Khaled Gohary with data analysis is gratefully acknowledged. The authors thank Daniel Myers, Justin Tindall, and Abraham Mendoza of the University of California Sierra Foothill Research and Extension Center for use of cattle, facilities, and assistance during completion of this study and Dr. Tor Tosteson for providing the blocked randomization scheme.

Open Access This article is distributed under the terms of the Creative Commons Attribution Noncommercial License which permits any noncommercial use, distribution, and reproduction in any medium, provided the original author(s) and source are credited.

\section{References}

Angelos JA, Ball LM (2007) Relatedness of cytotoxins from geographically diverse isolates of Moraxella bovis. Vet Microbiol 124, 382-386.

Angelos JA, Ball LM, Hess JF (2007a) Identification and characterization of complete RTX operons in Moraxella bovoculi and Moraxella ovis. Vet Microbiol 125, 73-79.

Angelos JA, Bonifacio RG, Ball LM, Hess JF (2007b) Prevention of naturally occurring infectious bovine keratoconjunctivitis with a recombinant Moraxella bovis pilin-Moraxella bovis cytotoxin-ISCOM matrix adjuvanted vaccine. Vet Microbiol 125, 274-283.

Angelos JA, Hess JF, George LW (2001) Cloning and characterization of a Moraxella bovis cytotoxin gene. Am J Vet Res 62, 1222-1228. 
Angelos JA, Hess JF, George LW (2003) An RTX operon in hemolytic Moraxella bovis is absent from nonhemolytic strains. Vet Microbiol 92, 363-377.

Angelos JA, Hess JF, George LW (2004) Prevention of naturally occurring infectious bovine keratoconjunctivitis with a recombinant Moraxella bovis cytotoxin-ISCOM matrix adjuvanted vaccine. Vaccine 23, 537-545.

Angelos JA, Spinks PQ, Ball LM, George LW (2007c) Moraxella bovoculi sp. nov., isolated from calves with infectious bovine keratoconjunctivitis. Int J Syst Evol Microbiol 57, 789-795.

Annuar BO, Wilcox GE (1985) Adherence of Moraxella bovis to cell cultures of bovine origin. Res Vet Sci 39, 241-246.

Beard MK, Moore LJ (1994) Reproduction of bovine keratoconjunctivitis with a purified haemolytic and cytotoxic fraction of Moraxella bovis. Vet Microbiol 42, 15-33.

Dueger EL, George LW, Angelos JA, Tankersley NS, Luiz KM, Meyer JA, Portis ES, Lucas MJ (2004) Efficacy of a long-acting formulation of ceftiofur crystalline-free acid for the treatment of naturally occurring infectious bovine keratoconjunctivitis. Am J Vet Res 65, 1185-1188.

Elad D, Yeruham I, Bernstein M (1988) Moraxella ovis in cases of infectious bovine keratoconjunctivitis (IBK) in Israel. Zentralbl Veterinarmed [B] 35, 431-434.

Fairlie G (1966) The isolation of a haemolytic Neisseria from cattle and sheep in the North of Scotland. Vet Rec 78, 649-650.

Funk L, O'Connor AM, Maroney M, Engelken T, Cooper VL, Kinyon J, Plummer P (2009) A randomized and blinded field trial to assess the efficacy of an autogenous vaccine to prevent naturally occurring infectious bovine keratoconjunctivis (IBK) in beef calves. Vaccine 27, 4585-4590.

George LW, Angelos JA, Ruehl WW (2004) Stability, antigenicity, and aggregation of Moraxella bovis cytolysin after purification and storage. Am J Vet Res 65, 977-983.

George LW, Borrowman AJ, Angelos JA (2005) Effectiveness of a cytolysin-enriched vaccine for protection of cattle against infectious bovine keratoconjunctivitis. Am J Vet Res 66, 136-142.

Gray JT, Fedorka-Cray PJ, Rogers DG (1995) Partial characterization of a Moraxella bovis cytolysin. Vet Microbiol 43, 183-196.

Henson JB, Grumbles LC (1960) Infectious Bovine Keratoconjunctivitis. I. Etiology. Am J Vet Res 21, 761766.

Hess JF, Angelos JA (2006) The Moraxella bovis RTX toxin locus mbx defines a pathogenicity island. J Med Microbiol 55, 443-449.

Hoien-Dalen PS, Rosenbusch RF, Roth JA (1990) Comparative characterization of the leukocidic and hemolytic activity of Moraxella bovis. Am J Vet Res 51, 191-196.

Ji GE, O'Hanley P (1990) Epitopes of Escherichia coli alpha-hemolysin: identification of monoclonal antibodies that prevent hemolysis. Infect Immun 58, 3029-3035.

Kagonyera GM, George L, Miller M (1989a) Effects of Moraxella bovis and culture filtrates on ${ }^{51} \mathrm{Cr}$-labeled bovine neutrophils. Am J Vet Res 50, 18-21.

Kagonyera GM, George LW, Munn R (1989b) Cytopathic effects of Moraxella bovis on cultured bovine neutrophils and corneal epithelial cells. Am J Vet Res 50, 10-17.

Lepper AW (1988) Vaccination against infectious bovine keratoconjunctivitis: protective efficacy and antibody response induced by pili of homologous and heterologous strains of Moraxella bovis. Aust Vet J $65,310-316$.

Moore LJ, Rutter JM (1989) Attachment of Moraxella bovis to calf corneal cells and inhibition by antiserum. Aust Vet J 66, 39-42.

Pugh GW, Jr., Hughes DE (1971) Infectious bovine keratoconjunctivitis induced by different experimental methods. Cornell Vet 61, 23-45.

Ruehl WW, Marrs C, Beard MK, Shokooki V, Hinojoza JR, Banks S, Bieber D, Mattick JS (1993a) Q pili enhance the attachment of Moraxella bovis to bovine corneas in vitro. Mol Microbiol 7, 285-288.

Ruehl WW, Marrs CF, George L, Banks SJ, Schoolnik GK (1993b) Infection rates, disease frequency, pilin gene rearrangement, and pilin expression in calves inoculated with Moraxella bovis pilin-specific isogenic variants. Am J Vet Res 54, 248-253.

Spradbrow PB (1967) A microbiological study of bovine conjunctivitis and keratoconjunctivitis. Aust Vet J $43,55-58$.

Tryland M, Das Neves CG, Sunde M, Mork T (2009) Cervid herpesvirus 2, the primary agent in an outbreak of infectious keratoconjunctivitis in semidomesticated reindeer. J Clin Microbiol 47, 3707-3713.

Weyant RS, Wayne Moss C, Weaver RE, Hollis DG, Jordan JG, Cook EC, Daneshvar MI (1996) Identification of Unusual Pathogenic Gram-Negative Aerobic and Facultatively Anaerobic Bacteria. 2 Edn. Williams and Wilkins, Baltimore.

Wilcox GE (1970) Bacterial flora of the bovine eye with special reference to the Moraxella and Neisseria. Aust Vet J 46, 253-256. 